\title{
C-erbB-2 expression is a better predictor for survival than galectin-3 or p53 in early-stage breast cancer
}

\author{
ANGELA F. LOGULLO ${ }^{1}$, ANDREIA B.G. LOPES ${ }^{2}$, SUELY NONOGAKI ${ }^{3}$, FERNANDO A. SOARES ${ }^{4}$, \\ MáRIO MOURÃO NETTO $^{5}$, INÊS N. NISHIMOTO ${ }^{6}$ and M. MITZI BRENTANI ${ }^{7}$
}

\begin{abstract}
${ }^{1}$ Departamento de Patologia da Universidade Federal de São Paulo; ${ }^{2}$ Hospital do Câncer; ${ }^{3}$ Departamento de Patologia do Instituto Adolfo Lutz; ${ }^{4}$ Departamento de Anatomia Patológica do Hospital do Câncer; ${ }^{5}$ Departamento de Mastologia do Hospital do Câncer; ${ }^{6}$ Setor de Epidemiologia e Bioestatística do Hospital do Câncer; ${ }^{7}$ Departamento de Radiologia, Faculdade de Medicina da Universidade de São Paulo (LIM-24), São Paulo, Brazil
\end{abstract}

Received January 19, 2007; Accepted March 5, 2007

\begin{abstract}
The definition of high risk patients with early stage breast cancer is still controversial. We evaluated the ability of galectin-3, c-erbB-2 and p53 immunohistochemical expression to predict recurrence and survival in a homogeneous set of 92 patients with T1N0M0 ductal carcinoma with a long-term follow-up. In normal breast tissue, the epithelial and fibroblast components were positive for galectin-3 mostly showing nuclear and cytoplasmic reactivity. At the tumor epithelial component, galectin-3 expression was found in $46.7 \%$ of the samples with a predominant cytoplasmic staining. Similar results were presented by concurrent in situ lesions. Tumor stromal fibroblasts maintained positivity in 70 out of 92 cases (76\%). We found expression of p53 in only 16 cases (17.4\%), and c-erbB-2 in 17 (18.48\%). A marginal association was found between co-expression of p53 and galectin- $3(\mathrm{p}=0.055)$ and a significant correlation between p53 accumulation and c-erbB-2 expression ( $\mathrm{p}=0.009)$. There was no significant association between galectin-3 protein expression with diseasefree survival or overall survival. C-erbB2 and p53 expression correlated with recurrence $(\mathrm{p}=0.002, \mathrm{p}=0.02$; respectively). Diminished overall survival at 10 years was associated with c-erbB-2 ( $\mathrm{p}=0.010)$, but marginally with p53 expression $(p=0.076)$. Epithelial galectin-3 expression cannot be considered a prognostic factor for patients with T1N0M0 breast cancer, p53 seems to be of minor relevance and c-erbB-2 expression was the best discriminator and may be a marker for aggressive clinical behavior in patients with early stage breast cancer.
\end{abstract}

Correspondence to: Dr M.M. Brentani, Faculdade de Medicina da USP, Departamento de Radiologia, Disciplina de Oncologia, Av. Dr. Arnaldo, $455,4^{\circ}$ andar, sala 4112, São Paulo, Brazil E-mail: mbrentani@lim24.fm.usp.br

Key words: breast carcinoma, T1N0M0, c-erbB-2, p53, galectin-3, immunohistochemistry, survival, disease-free survival, prognosis

\section{Introduction}

Breast cancer is the most common malignant tumor and the second leading cause of cancer mortality in women. The progressive improvement in breast cancer mortality is largely due to detection of early stage carcinomas and pre-malignant lesions (1). Patients with such an early diagnosed breast cancer tend to have good survival but a small percentage of them die of their disease. Although effective for large tumors, most classical prognostic factors are less reliable for small tumors and further stratification of these patients remains a challenge (2-5). Thus, prognostic factors to identify the high-risk group among these patients are needed.

On the early set of invasion, malignant transformation is often accompanied by a variety of changes in cell-cell and cell-matrix interactions which could lead to abnormal cell behaviour. Galectins are a family of non-integrin B-galactoside binding lectins with related amino acid sequences defined by structural similarities in their carbohydrate-binding domains. An important member of this family, galectin-3 is a chimeric type of galectin which has been implicated in diverse biological functions in normal and pathophysiological conditions (6). In breast carcinomas, galectin-3 expression is usually decreased when compared with their respective normal counterparts and the reduced expression in epithelial cells is associated with advanced histological grade $(7,8)$. Such results suggested that galectin-3 expression seems to be gradually decreased following the tumor progression.

Some histological factors have been described that identify patients with stage I breast cancer with a relatively worse prognosis (5). Few studies analyzed the overexpression of p53 and erbB-2 as predictors for recurrence and survival in early breast cancer (9-12). Galectin-3 has been associated with c-erbB-2 expression and down-regulated by p53 $(13,14)$. Investigations of the relevance of galectin expression as prognostic factors in breast cancer have been limited $(15,16)$.

In the present study, to better understand the association between galectin-3 and clinical outcome in invasive ductal carcinoma we measured the immunohistochemical expression of galectin-3 along the c-erbB-2 and p53 expression in the 
tumors of a homogeneous set of stage I invasive ductal carcinoma patients with long-term clinical follow-up. We then compared the prognostic significance of these markers in terms of relapse-free survival and overall survival of the patients.

\section{Patients and methods}

We studied a retrospective sequential group of paraffinembedded samples of infiltrating ductal carcinomas clinically classified as T1N0M0; collected, diagnosed and treated at Hospital do Câncer A.C. Camargo, São Paulo, between 1993 and 1994. Ninety-two cases were included, all with slides and suitable paraffin blocks for immunohistochemical analysis, clinical and survival data available. Conventional clinicopathological features of each case including tumor size, histologic grade and lymph node status, presence of recurrence, occurrence of death caused by cancer or other causes were reviewed. All cases were classified according to the TNM (tumor, node, metastasis) classification system (UICC). Slides were reviewed in order to confirm diagnosis. Histological grade was established according to the Notthingham system (17). Age varied from 30 to 86 years, with a median of 56 years; 25 patients were pre-menopausal and 67 postmenopausal. Only two patients indicated a family history of breast cancer. Treatment protocols were homogeneous due to protocol adherence. All patients were submitted to surgery and lymphadenectomy. Surgical approach varied from quadrantectomy to sectorectomy. Regional and axillary lymph nodes were ressected and evaluated in all cases. Postoperative adjuvant radiation therapy was administered to all patients. Adjuvant therapy was performed as chemotherapy with cyclophosphamide, methotrexate and 5fluorouracil (CMF) in 20 patients. Hormone therapy was performed with tamoxifen in 32 patients. This investigation was performed after approval by the Hospital do Câncer review board.

Immunohistochemical methods. Antigens p53 and galectin-3 were assessed with the following antibodies: c-erb-2 (Dako, Inc.) p53 antibody (DO-7), galectin-monoclonal antibody M3/38 TIB166 (ATCC, Manassas, VA, USA).

Positive and negative control slides were used in all reactions. We used previously known female breast cancer cases as positive controls. Negative controls were performed by incubating slides with PBS instead of primary antibody.

Briefly, after deparaffinization in xylol and alcohol, slides (3- $\mu \mathrm{m}$ thick) were immersed in PBS, $\mathrm{pH} \mathrm{7.4,} \mathrm{for} 5 \mathrm{~min}$ and submitted to microwave treatment for epitope retrieval with citric acid solution, pH 6.0, with two 9-min cycles. The material was submitted to endogenous peroxidase blocking treatment $\left(10\right.$ vol. $\left.\mathrm{H}_{2} \mathrm{O}_{2}\right)$ followed by another PBS immersion, and by overnight incubation in a humid chamber at $4^{\circ} \mathrm{C}$ with the primary antibody at $1 / 50$ dilution for $p 53,1 / 16$ for galectin- 3 and $1 / \mathrm{X}$ for $\mathrm{c}$-erbB-2. Slides were then rinsed in PBS solution and incubated with the biotinylated secondary antibody (1:200 dilution; Dako) for $30 \mathrm{~min}$ and again rinsed and incubated with the ancillary antibody (StrepAB complex/ HRP Duet, mouse/rabbit) for another $30 \mathrm{~min}$. The material was developed by immersion in 2,3 diaminobenzidine tetrahydrochloride (DAB, Sigma Chemical Co., St. Louis, MO,
USA), $60 \mathrm{mg} \%+1 \%$ dimethyl sulfoxide at $37^{\circ} \mathrm{C}$ for $5 \mathrm{~min}$ and by hematoxylin counterstaining. Positivity was characterized by dark brown staining of nucleus (p53) or cell membrane (c-erbB-2) or cell membrane and cytoplasm (galectin-3). A case was considered positive when $>10 \%$ of tumor cells were stained.

Statistical analysis. The Chi-square test was used to assess the univariate association of clinicopathological characteristics with p53, and galectin- 3 and c-erbB- 2 in $2 \times 2$ tables. When at least one variable expected was $<5 \%$, the Fisher's exact test was performed. The overall threshold of significance level was 0.5 . To calculate the survival rate and the survival curves the time gap between the date of surgery and the last contact available (for the majority living patients) or the date of death was used. Patients who died of causes other than breast cancer $(n=3)$ were treated as non-censored observations. The overall and disease-free survival was estimated using the Kaplan-Meier method. The log-rank test was used to compare survival curves with 5\% level of significance.

\section{Results}

Pathological variables. All cases were classified as T1, and more specifically 15 as T1a, 30 as T1b and 47 as T1c. Axillary lymph nodes were positive in only 2 cases. In all the other 90 cases, no neoplastic involvement was identified. By the time of surgery no patient presented metastasis. Among the 92 invasive ductal carcinomas included in this series, 20 were categorized as histological grade I, 45 as grade II and 27 as grade III. Twenty-seven samples presented an 'in situ' component associated to the invasive tumor. Expression of estrogen and progesterone receptors was detected in 52 and 35 cases, respectively.

Galectin-3 expression. In 66 out of 92 samples, there was enough adjacent normal breast tissue to be evaluated and comparing galectin-3 expression to the pattern occurring in the neoplastic tissue. The normal breast epithelia adjacent to tumor area were usually positive to galectin-3, exhibiting a moderate intensity. The distribution pattern was mostly of double staining (nuclear and cytoplasmic positivity), identified in 54 cases $(81.82 \%)$; but exclusive nuclear pattern was reported in 8 samples $(12.12 \%)$ and exclusive cytoplasmatic staining was observed in only 4 cases $(6.06 \%)$. Fig. 1A shows that galectin-3 is present in normal breast epithelium. Normal stromal cells, evaluated at the same samples when non-malignant adjacent tissue was available (64 samples), showed an exclusively nuclear pattern of galectin-3 expression in the majority of cases (96.9\%); (Fig. 1B). Within the entire tumor population, positive galectin expression, found in 43 tumors $(46.7 \%)$ was characterized by frequent localization in the cytoplasm ( $65 \%$ of the positive samples) (Fig. 1C). The remaining positive cases showed a simultaneous of cytoplasmatic/nuclear staining (Fig. 1D). The staining intensity was comparable or less strong than the normal breast pattern, and histological grade I, II and III showed positivity for galectin-3 in 40, 55 and $37 \%$ of lesions, respectively, suggesting a slight tendency for decreased positivity in grade III. On the other hand, more than half of 

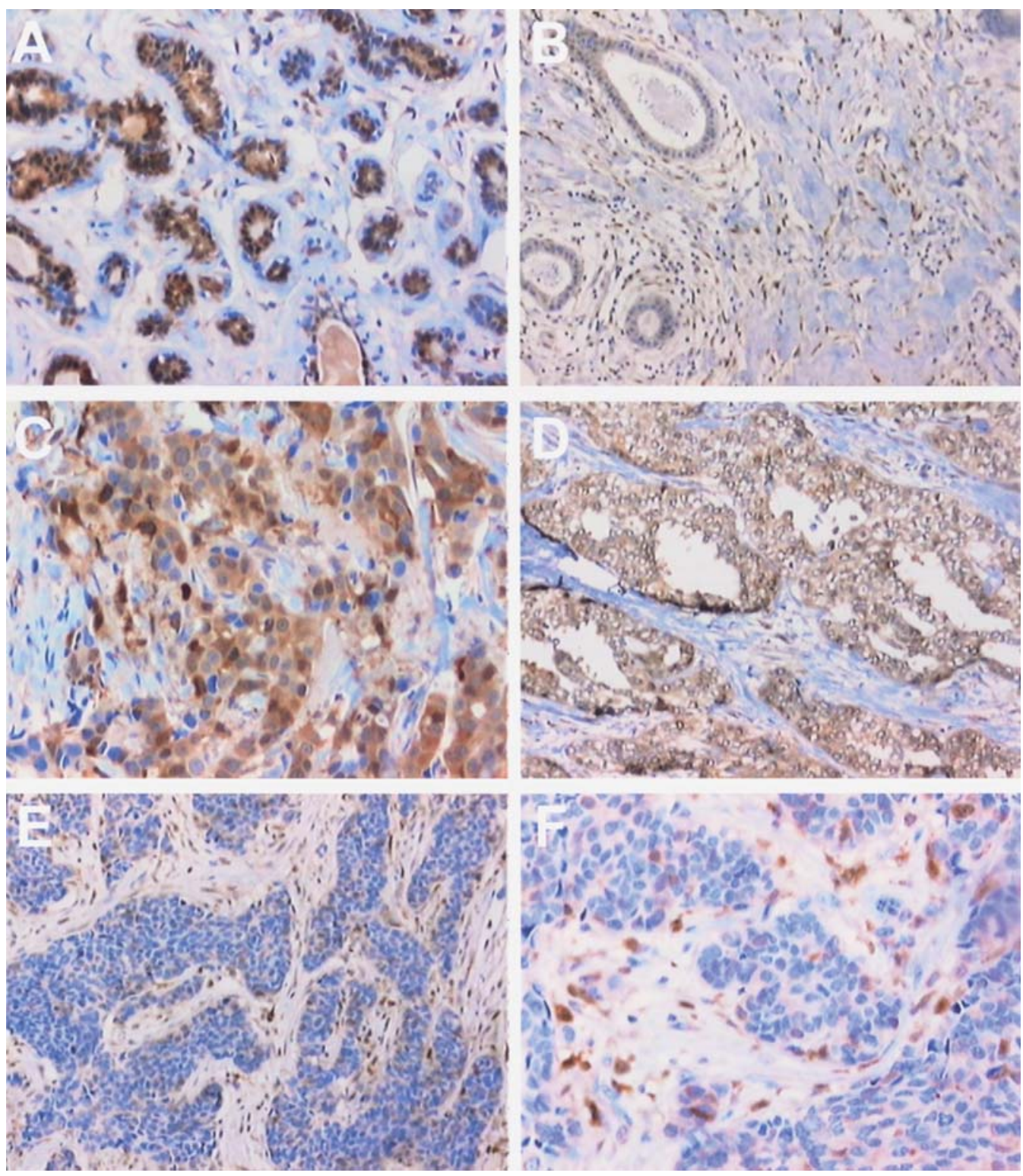

Figure 1. Immunohistochemical galectin-3 staining of breast cancer (T1NOM0) samples illustrating cellular distribution: (A) normal breast lobule (x200); (B) positive stromal reaction in normal breast tissue (x100); (C) ductal carcinoma showing double positive staining (x400); (D) ductal carcinoma showing cytoplasmic reactivity (x200); (E) positive tumor stromal reaction (x200); (F) negative staining in the tumor sample but positive stromal reaction (x400).

tumors did not show epithelial expression of galectin-3 (49 cases, $53.36 \%$ ); (Fig. 1F). We also analyzed the presence of galectin-3 expression at the breast stromal component. Galectin-3 expression at the tumor stromal fibroblast cells was commonly observed in 70 out of 92 cases $(76.09 \%)$ that exhibited a exclusive nuclear positivity, with scanty cytoplasmatic staining (Fig. 1E). No staining was observed in the remaining 22 cases $(23.91 \%)$. Expression in the tumor epithelial and stromal components was not always coincident. We also analyzed the galectin-3 expression at the in situ component of the 27 cases containing concurrent ductal carcinoma 'in situ' and the invasive ductal component; galectin-3 was negative in 16, cytoplasmic positivity was present in 13 and double staining was verified in 4 samples.

p53 and c-erbB-2 expression. Expression of p53 was detected exclusively in the nucleus of epithelial neoplastic epithelial cells in a strong and homogeneous pattern in only 16 cases (17.4\%), all the remaining 76 cases were negative. C-erbB-2 was expressed in 17 (18.5\%) cases and negative in 75 (81.5\%) Normal adjacent epitelia were always negative for p53 and c-erbB-2 expression.

A marginal significant association was found between positive staining for galectin-3 and $\mathrm{p} 53$. Among the 16 positive p53 cases, 12 (75\%) were simultaneously galectin-3 positive, whereas among the p53 negative cases (76 cases), 31 (40.8\%) were galectin- 3 positive $(p=0.055)$. No association was found between c-erbB-2 and galectin-3 $(\mathrm{p}=0.215)$. The $\mathrm{p} 53$ expression often occured together with c-erbB-2 expression in the same tumor $(\mathrm{p}=0,009)$. Among the 16 positive cases for p53, 7 were also positive for c-erbB-2. At the in situ component of the tumor, expression of galectin-3, p53 and c-erbB-2 was commonly coincident with the expression observed in the invasive component $(\mathrm{p}<0.05)$. 

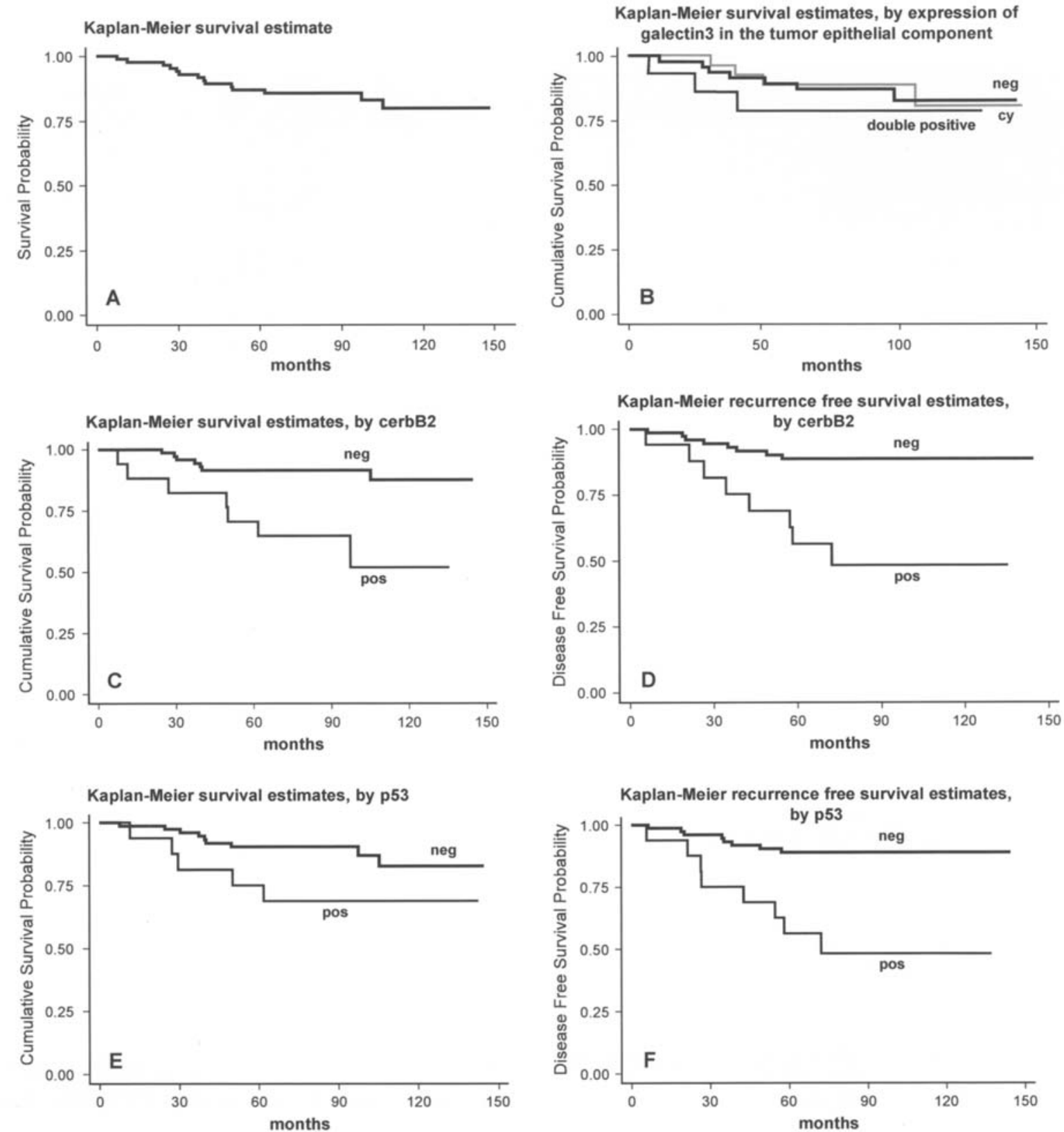

Figure 2. Kaplan-Meier survival estimate (months): (A) five- and 10-year overall survival and according to; (B) expression of galectin-3 in the tumor epithelial components; negative (neg), positive in the cytoplasmic (cy, grey line) and showing double positivity (cytoplasmic plus nucleus); (C) c-erbB-2 positive and negative; (E) p53 positive and negative; (D and F) recurrence-free survival respectively for patients with c-erbB-2 and p53 negative or positive.

Correlation to outcome. The median follow-up was 84.0 months. At the last follow-up (November 2005), $11(12 \%)$ patients were deceased, 8 (9\%) were alive with controlled disease, and $66(72 \%)$ were alive without disease. Recurrence was detected in $14(15 \%)$ of the patients and 4 were lost to follow-up. Fig. 2 depicts the Kaplan-Meier plot of overall and disease-free survival rates. The five and 10 years overall survival was $87.5 \%$ and $80.5 \%$, respectively (Fig. $2 \mathrm{~A}$ ).

Galectin-3 expression in the epithelial component was analyzed in relation to survival parameters. No significant association was evident between galectin-3 protein expression in terms of overall survival (Fig. 2B). Patients whose tumors exhibited positive cytoplasmic galectin-3 expression had a survival rate of $88.9 \%$ at five years and $80.8 \%$ at ten years. Similar rates were found for galectin-3 negative tumors being respectively $89.4 \%$ and $81.9 \%$. For tumors exhibiting double positivity (cytoplasm plus nucleus) patients had survival rates of $78.8 \%$ at five and $76.0 \%$ at ten years, respectively (logrank test, $\mathrm{p}=0.6780$ ). Galectin-3 expression did not show any association with recurrence-free survival for comparison among curves of double positive, negative and cytoplasmic staining (log-rank test, $\mathrm{p}=0.1348$; data not shown).

C-erbB-2 expression correlated with overall survival $(p=0.010$, Fig. $2 \mathrm{C})$ and with recurrence ( $\mathrm{p}=0.002$, Fig. $2 \mathrm{D})$. Although p53 expression similarly to c-erbB-2 was associated with recurrence ( $\mathrm{p}=002$, Fig. $2 \mathrm{~F}$ ) it reached only a marginal correlation with survival ( $\mathrm{p}=0.076$, Fig. 2E).

\section{Discussion}

In the present study, we analyzed galectin- 3 expression as well as its sub-cellular distribution in a homogeneous set of 
early stage breast cancer to examine the possible involvement of galectin-3 in progression of these tumors. In our series, $\sim 46.6 \%$ of 92 invasive carcinomas were positive for galectin-3 estimating only the epithelial constituent, a similar percentage to that reported $(49.4 \%)$ for a heterogeneous series of primary breast cancer tumors, including only $22 \%$ of stage I samples (16). Although not statistically significant, loss of galectin-3 expression tended to increase in high grade samples, agreeing with previous results of immunohistochemical evaluation of galectin-3 in invasive breast cancers (8). Our observation that in the lesions containing 'in situ' with concurrent invasive carcinomas, the galectin-3 positivity of both components was similar, suggested that galectin-3 loss is already observed in early steps of transformation. Previous work has shown that when synchronous invasive and 'in situ' cancer coexist, both components are likely to share virtually identical genomic changes (18). Our results conflict with previous studies describing preserved expression of galectin-3 in carcinoma 'in situ' (8). However, it is possible that pure DCIS expresses higher levels of galectin-3 and constitutes a distinct lesion from DCIS associated to invasive ductal carcinoma.

On the contrary, transcripts of galectin-3 gene were detected in $78.4 \%$ of 106 breast cancer samples evaluated by O'Driscoll et al (15) and their series included $18 \%$ of T1 cases. The galectin-3 production by fibroblasts could possible explain these results as galectin-3 mRNA total levels may reflect the stromal content (19). A recent study observed galectin-3 expression in tumor cells that are in close proximity to the stroma in a breast cancer xenograft model (20). These contradictory conclusions may result from the fact that invasive breast cancer cells may acquire mesenchymal characteristics, losing epithelial features through cancer progression.

Our results on the cellular localization of galectin-3 are in agreement with previous observations in several other types of carcinomas showing that galectin-3 immunoreactivity in contrast to non-tumoral epithelial cells is frequently present in the cytoplasm (21-24). The nuclear localization of galectin-3 is presumably associated with normal regulation of cell proliferation and apoptosis since it is a required splicing factor interacting with Gemini 4 (25). It was also postulated that in an experimental prostate cancer model system, nuclear galectin-3 suppressed malignancy whereas cytoplasmic galectin-3 promoted tumorigenicity (21). In the cytoplasmic compartment galectin-3 may act as an anti-apoptotic factor (26). The cytoplasmic/nuclear distribution seems to be regulated by multiple process not yet completely characterized (27).

Given the potential involvement of galectin-3 subcellular localization in progression we rationalized that cytoplasmic galectin-3 might be an indicator of clinical outcome. However, our analysis of the survival curves leads to the conclusion that galectin-3 expression, regardless of the tumor cellular localization, is not a suitable marker to evaluating risk in T1N0M0 breast carcinomas. Jones et al (16) correlated galectin-3 nuclear expression of primary breast tumors (2\% of the cases) with a shorter overall survival. As primary tumors are heterogeneous we can not rule out that the emergence of a minority of sub-clones localized in the nucleus may favor tumor progression. O'Driscoll et al (15) have previously suggested that galectin-3 mRNA could not be considered a prognostic indicator of disease outcome. However, they have not taken into account that the measured galectin- 3 expression might be influenced by the stromal component.

p53 cannot be considered a prognostic indicator of disease outcome for patients with T1N0M0 breast cancer. However, c-erb-2 expression correlated to outcome variables, recurrence and survival in univariate analysis and thus may be a marker for aggressive clinical behaviour in patients with early stage breast cancer confirming previous results (reviewed in ref. 5).

\section{Acknowledgements}

This study was supported by FAPESP 04/04607-8 and CNPq.

\section{References}

1. Ernster VL, Ballard-Barbash R, Barlow WE, Zheng Y, Weaver DL, Cutter G, Yankaskas BC, Rosenberg R, Carney PA, Kerlikowske K, Taplin SH, Urban N and Geller BM: Detection of ductal carcinoma in situ in women undergoing screening mammography. J Natl Cancer Inst 94: 1546-1554, 2002.

2. Tabar L, Duffy SW, Vitak B, Chen HH and Prevost TC: The natural history of breast carcinoma: what have we learned from screening? Cancer 86: 449-462, 1999.

3. Joensuu H, Pylkkanen L and Toikkanen S: Late mortality from pT1N0M0 breast carcinoma. Cancer 85: 2183-2189, 1999.

4. Westenend PJ, Meurs CJ and Damhuis RA: Tumour size and vascular invasion predict distant metastasis in stage I breast cancer. Grade distinguishes early and late metastasis. J Clin Path 58: 196-201, 2005.

5. Hanrahan EO, Valero V, Gonzalez-Angulo AM and Hortobagyi GN: Prognosis and management of patients with node-negative invasive breast carcinoma that is $1 \mathrm{~cm}$ or smaller in size (stage 1; T1a,bN0M0): a review of the literature. J Clin Oncol 24: 2113-2122, 2006.

6. Liu FT and Rabinovich GA: Galectins as modulators of tumour progression. Nat Rev Cancer 5: 29-41, 2005.

7. Castronovo V, Van Den Brule FA, Jackers P, Clausse N, Liu FT, Gillet $\mathrm{C}$ and Sobel ME: Decreased expression of galectin-3 is associated with progression of human breast cancer. J Pathol 179: 43-48, 1996.

8. Idikio $\mathrm{H}$ : Galectin-3 expression in human breast carcinoma: correlation with cancer histologic grade. Int J Oncol 12: 1287-1290, 1998.

9. Joensuu H, Isola J, Lundin M, Salminen T, Holli K, Kataja V, Pylkkanen L, Turpeenniemi-Hujanen T, von Smitten K and Lundin J: Amplification of erbB2 and erbB2 expression are superior to estrogen receptor status as risk factors for distant recurrence in pT1N0M0 breast cancer: a nationwide populationbased study. Clin Cancer Res 9: 923-930, 2003.

10. Linjawi A, Kontogiannea M, Halwani F, Edwardes $\mathbf{M}$ and Meterissian S: Prognostic significance of p53, bcl-2, and Bax expression in early breast cancer. J Am Coll Surg 198: 83-90, 2004.

11. Lee WY, Su WC, Lin PW, Guo HR, Chang TW and Chen HH: Expression of S100A4 and Met: potential predictors for metastasis and survival in early-stage breast cancer. Oncology 66: 429-438, 2004.

12. Colleoni M, Rotmensz N, Peruzzotti G, Maisonneuve P, Viale G, Renne G, Casadio C, Veronesi P, Intra M, Torrisi R and Goldhirsch A: Minimal and small size invasive breast cancer with no axillary lymph node involvement: the need for tailored adjuvant therapies. Ann Oncol 15: 1633-1639, 2004.

13. Gaudin JC, Arar C, Monsigny M and Legrand A: Modulation of the expression of the rabbit galectin-3 gene by p53 and c-Ha-ras proteins and PMA. Glycobiology 7: 1089-1098, 1997.

14. Mackay A, Jones C, Dexter T, Silva RL, Bulmer K, Jones A, Simpson P, Harris RA, Jat PS, Neville AM, Reis LF, Lakhani SR and O'Hare MJ: cDNA microarray analysis of genes associated with ERBB2 (HER2/neu) overexpression in human mammary luminal epithelial cells. Oncogene 22: 2680-2688, 2003. 
15. O'Driscoll L, Linehan RM, Kennedy S, Cronin D, Purcell R, Glynn SW, McDermott ED, Hill AJ, O'Higgins N, Parkinson M and Clynes M: Lack of prognostic significance of survivin, survivin-deltaEx3, survivin-2B, galectin-3, bag-1, bax-alpha and MRP-1 mRNAs in breast cancer. Cancer Lett 201: 225-236, 2003.

16. Jones C, Mackay A, Grigoriadis A, Cossu A, Reis-Filho JS, Fulford L, Dexter T, Davies S, Bulmer K, Ford E, Parry S, Budroni M, Palmieri G, Neville AM, O'Hare MJ and Lakhani SR: Expression profiling of purified normal human luminal and myoepithelial breast cells: identification of novel prognostic markers for breast cancer. Cancer Res 64: 3037-3045, 2004.

17. Elston CW and Ellis IO: Pathological prognostic factors in breast cancer. I. The value of histological grade in breast cancer: experience from a large study with long-term follow-up. Histopathology 19: 403-410, 1991.

18. van de Vijver MJ: Biological variables and prognosis of DCIS. Breast 14: 509-519, 2005.

19. Moiseeva EV, Rapoport EM, Bovin NV, Miroshnikov AI, Chaadaeva AV, Krasilshschikova MS, Bojenko VK, Bijleveld C, van Dijk JE and Den Otter W: Galectins as markers of aggressiveness of mouse mammary carcinoma: towards a lectin target therapy of human breast cancer. Breast Cancer Res Treat 91: 227-241, 2005.

20. Shekhar MP, Nangia-Makker P, Tait L, Miller F and Raz A: Alterations in galectin-3 expression and distribution correlate with breast cancer progression: functional analysis of galectin-3 in breast epithelial-endothelial interactions. Am J Pathol 165: 1931-1941, 2004.
21. Califice S, Castronovo V, Bracke M and van den Brule F: Dual activities of galectin-3 in human prostate cancer: tumor suppression of nuclear galectin-3 vs. tumor promotion of cytoplasmic galectin-3. Oncogene 23: 7527-7536, 2004.

22. Honjo $\mathrm{Y}$, Inohara $\mathrm{H}$, Akahani S, Yoshii T, Takenaka $\mathrm{Y}$, Yoshida J, Hattori K, Tomiyama Y, Raz A and Kubo T: Expression of cytoplasmic galectin-3 as a prognostic marker in tongue carcinoma. Clin Cancer Res 6: 4635-4640, 2000.

23. van den Brule FA, Waltregny D, Liu FT and Castronovo V: Alteration of the cytoplasmic/nuclear expression pattern of galectin-3 correlates with prostate carcinoma progression. Int J Cancer 89: 361-367, 2000.

24. Lotz MM, Andrews CW Jr, Korzelius CA, Lee EC, Steele GD Jr, Clarke A and Mercurio AM: Decreased expression of Mac-2 (carbohydrate binding protein 35) and loss of its nuclear localization are associated with the neoplastic progression of colon carcinoma. Proc Natl Acad Sci USA 90: 3466-3470, 1993.

25. Park JW, Voss PG, Grabski S, Wang JL and Patterson RJ: Association of galectin-1 and galectin-3 with Gemin 4 in complexes containing the SMN protein. Nucleic Acids Res 29: 3595-3602, 2001

26. Nakahara S, Oka N and Raz A: On the role of galectin-3 in cancer apoptosis. Apoptosis 10: 267-275, 2005.

27. Nakahara S, Oka N, Wang Y, Hogan V, Inohara H and Raz A: Characterization of the nuclear import pathways of galectin-3. Cancer Res 66: 9995-10006, 2006. 